Ann. Zootech., I975, $24(4), 66 \mathrm{r}-670$.

\title{
INDUCTION DE LA MISE BAS DE LA TRUIE PAR UN ANALOGUE DE LA PROSTAGLANDINE $\mathbf{F}_{2 \alpha}$ : CONSÉQUENCES ZOOTECHNIQUES
}

\author{
M. J. BOSC, F. MARTINAT-BOTTE* et P. DUCHENE \\ Station de Physiologie de la Reproduction, \\ Centre de Recherches de Tours, I. N.R. A., \\ Nouzilly, 37380 Monnaie, B.P. 1 \\ * Institut Technique du Porc, \\ 149, rue de Bercy, \\ 75579 Paris Cedex 12
}

\section{RÉSUMÉ}

L'administration intramusculaire d'un analogue de la l'rostaglandine $\mathrm{F}_{2 \alpha}$ ou A-PGF (composé ICI no 80996) induit la mise bas de la Truie. Ainsi l'intervalle "traitement-part " est égal à $29 \mathrm{~h} 44$

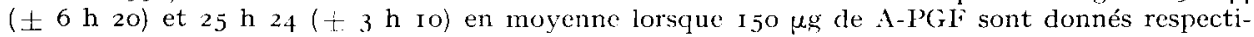
vement le I I $^{\circ}$ et le i I ${ }^{\mathrm{e}}$ jour de la gestation alors que pour les témoins les intervalles comparables sont de Ioo h et de $76 \mathrm{~h}(+35 \mathrm{~h})$. Ces intervalles sont liés ì la prolificité car la corrélation est égale à $-0,5^{6}$ pour toutes les truies traitées. La durée d'expulsion est très variable, de $153 \mathrm{mn}$

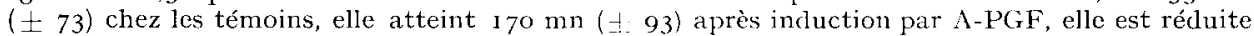
à I $24 \mathrm{mn}( \pm 70)$ lorsque deux injections d'ocytocine sont effectuées après la naissance du I er foetus.

Le poids de naissance est plus faible et la mortalité au cours des deux premiers jours de leur vie est plus élevée pour les porcelets des truies traitées par A-l'GF que pour les témoins. Par contre, la croissance des porcelets pendant la première semaine de lactation ne semble pas affectée lorsque A-PGF a été donnée le ir i jour de la gestation.

\section{INTRODUCTION}

Chez la Truie la chute du taux plasmatique de progestérone précède le part (Killian, Garverick et I)Ay, I972; MolokwU et Wagner, I973; STABenfeldt, I974) et lève ainsi l'inhibition exercée par cette hormone sur l'activité utérine (Rousseau et PRUD'homme, I974). Cette baisse de la progestéronémie est la conséquence de la régression des corps jaunes contrôlés par les fœetus eux-mêmes par l'intermédiaite d'une substance lutéolytique utérine (Bosc, du Mesnit du Busson 
et LocATtiL I, I 974). Cette dernière est vraisemblablement la prostaglandine $F_{\mathrm{g} \alpha}$ $\left(\mathrm{PGF}_{2 \alpha}\right)$ conme cela a été montré chez de nombreux mammifères au cours du cycle œestrien et en fin de gestation chez la Chèvre (Currif et Thorburn, I973) la Lapine (Nathanielsz, Abei et Smith, I972; Challis, Davies et Ryan, I973) ou la Rate (Aiken, I972 ; Dunn et al., I973; Buckiz et Nathanizisz, I973). Compte tenu de cette propriété lutéolytique, mais aussi de leur action sur l'activité utérine, les prostaglandines $F_{2}$ et $E_{12}$ ont été très utilisées pour provoquer l'avortement ou l'accouchement chez la Femme (Bygdeman et Wiovist, I974 ; 'THIERY, I974) et la mise bas chez d'autres mammifères (Bosc, I974). Ainsi chez la Truie le cochonnage est-il induit en $28 \mathrm{~h}$ par perfusion de $\mathrm{PGF}_{2 \alpha}$ (DIEHL et al., I973). La disponibilité d'analogues de synthèse de ces composés permet d'envisager un contrôle efficace et facile de la mise bas dans cette espèce. Avec un de ceux-ci, Ash et Heap (I973) ont provoqué le cochonnage en $28 \mathrm{~h}$. Au cours d'un essai préliminaire, nous avions aussi obtenu un résultat semblable sans noter d'effets secondaires sur la mortalité postnatale des porcelets ni sur leur croissance au cours de la première semaine de leur vie.

Afin de préciser les potentialités et les conséquences zootechniques d'un tel produit, nous avons entrepris l'expérience dont nous présentons les résultats.

\section{MATÉRIEL ET MÉTHODES}

Cette expérience a été réalisée sur des truies (Hybride, Landrace, Large-White) d'un élevage du centre de la. France. Les dates d'insémination, le jour et l'heure des mise bas ont été relevés. Trois lots ont été constitués. Dans le lot Prostaglandine (lot I'G) 29 truies multipares ont eu une seule administration intramusculaire de $\mathrm{I}_{50} \mu \mathrm{g}$ d'un analogue de $\mathrm{PGF}_{2 \alpha}$ (A-PGI) (composé ICI $\left.\mathrm{n}^{\circ} 80996\right)^{(1)}$ à 8 ou I I h le I Io ou le I I i jour de la gestation. Quatre truies supplémentaires ont reçu dans les mêmes conditions $I 00 \mu g$ du même prodiuit. Dans le deuxième lot (Lot ()), 20 truies ont subi un traitement assez souvent utilisé en élevage : après l'expulsion du premier porcelet et une heure après environ, chaque animal a eu une injection de $5 \mathrm{ml}$ d'une solution (Intertocine) contenant l'équivalent de ro UI d'ocytocine par ml. Vingt truies contemporaines n'ont subi aucun traitement; elles forment le lot témoin (Lot T).

Les jours et les heures de traitement et d'expulsion des porcelets ont été notés. L'état et le poids des porcelets de chaque portée ont été relevés à la naissance et au $7^{\mathbf{e}}$ jour de leur vie et au $\mathrm{I}_{-2^{2}}$ et $3^{\mathrm{e}}$ jour après la naissance pour tontes les truies des lots $T$ et $O$ et pour 20 truies du lot PG ( 13 traitées au i I ${ }^{\mathrm{e}}$ jour et 7 au I Io $^{\mathrm{e}}$ ).

Les analyses ont porté sur les intervalles "traitement-parturition " des truies dont la mise bas a été induite par l'analogue de $\mathrm{PGF}_{2 \alpha}$ (A-PGF), sur les durées d'expulsion des porcelets, et sur la croissance (Covariance) et sur la mortalité des porcelets des différents lots (Test $\chi^{2}$ de Pearson).

\section{RÉSULTATS}

Dans l'élevage considéré, les mise bas se répartissent du I IO $^{\mathrm{e}}$ au $\operatorname{II} 9^{\text {e jour de }}$ la gestation (observations de I 022 gestations). Au moment où les truies ont reçu l'analogue de $\mathrm{PFG}_{2 \alpha}$ (A-PGF), il n'y a normalement que 2,3 p. Ioo des mise bas $\left(0,7\right.$ p. roo le Iroe et I,6 p. Ioo le II I ${ }^{\text {e }}$ jour de la gestation) ; 5,9 p. Ioo se produisent le $\operatorname{Ir} 2^{e}$ jour.

(1) Nous remercions "Imperial Chemical Industries" (U. K.) d'avoir aimablement mis à notre disposition ce produit. 
Les truies traitées par $150 \mu \mathrm{g}$ d'A-GPF le I I $^{\mathrm{e}}$ jour de la gestation ont mis bas 29 h 44 ( \pm 6 h 19 ) en moyenne après le traitement, celles qui ont été traitées le

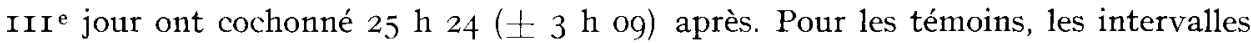
comparables calculés entre le I ro $^{\mathbf{e}}$ ou le I I I ${ }^{\mathrm{e}}$ jour de gestation et le terme moyen sont respectivement de Ioo h et $76 \mathrm{~h}$ avec une variabilité de $35 \mathrm{~h}$ (tabl. I). Sur la figure I, les intervalles "traitement-part " sont présentés en fonction de la taille de la portée. Il existe en effet une relation entre ces deux paramètres : elle semble dépendre du jour de traitement. Ainsi la corrélation entre l'intervalle " traitement-part " et la prolificité est égale à - 0,56 pour toutes les truies qui ont eu $\mathrm{I}_{5} 0 \mu \mathrm{g}$ d'A-PGF (tabl. I, fig. I).

\section{TABLEAU I}

Intervalles "traitement-parturition " des truies recevant (I.M.) un analogue de $P G F_{2 \alpha}\left(I C I n^{\circ} 80996\right)$

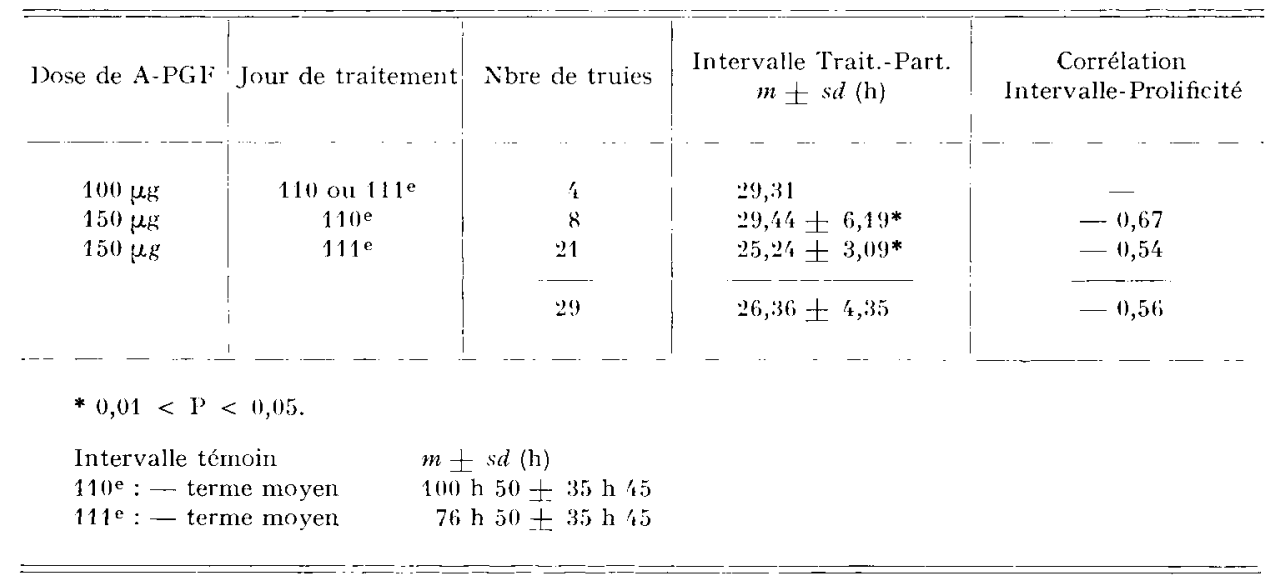

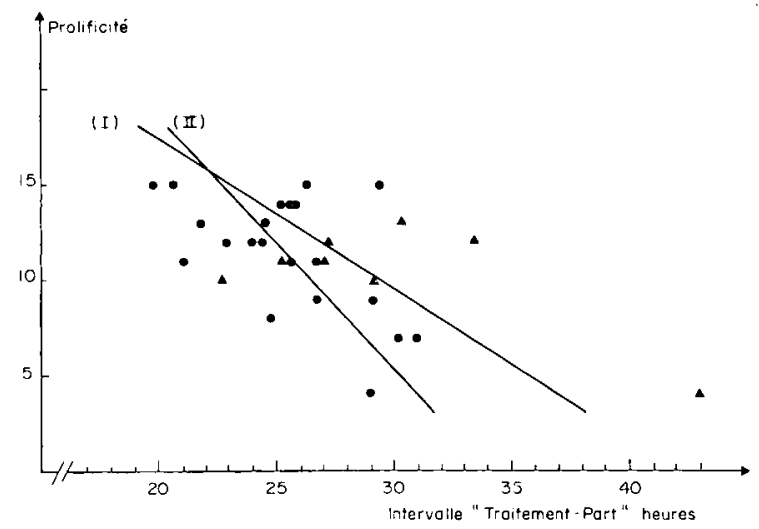

FIG. I. - Intervalle "Traitement-Part " et prolificité des truies traitêes par un analogue de $P G F_{2 \alpha}$ (I.C.I. ${ }_{\mathrm{I} 1}^{\circ} 80996$ )

(I) - Truies traitées au I IO $^{\mathrm{C}}$ ol au I I I jour de gestation $(y=-0,37 x+2 \mathrm{I}, 06)$

(II)•Truies traitées au I I I jour de gestation $(y=-0,54 \times 1 \quad 25,2 \mathrm{I})$ 
Il faut noter que 26 des 29 truies traitées ont mis bas 20 à $32 \mathrm{~h}$ après le traitement c'est-à-dire entre $7 \mathrm{~h}$ et Ig h le lendemain du traitement d'induction (fig. I). On augmente de cette manière les mise bas au cours de la période claire; en effet dans l'élevage considéré, Io mois d'observations ont montré que sur 895 cochonnages 69 p. roo d'entre eux se produisaient entre ces deux heures de la journée.

Avec $100 \mu \mathrm{g}$ d'A-PGF la mise bas s'est produite en moyenne $29 \mathrm{~h}$ après l'injection ce qui suggère que cette dose pourrait être suffisante pour induire le cochonnage (tabl. I).

Les expulsions des porcelets ont été suivies sur 59 truies : chez les truies témoins la durée moyenne d'expulsion de tous les fotus est égale à I53 $\mathrm{mn}$ ( $\pm 73 \mathrm{mn}$ ), elle est différente de celles des truies induites par A-PGF (I70 mn \pm 93) et des truies recevant 1'ocytocine (123 mn \pm 70). La corrélation entre la durée d'expulsion et la taille de la portée est pratiquement nulle $(-0,06)$ pour les animaux des lots $\mathrm{T}$ et $\mathrm{PG}$ ce qui indique une absence de relation entre ces deux paramètres. Administrer de l'ocytocine raccourcit la durée moyenne d'expulsion et augmente cette corrélation qui est alors égale à $0, \mathbf{I} 80$ (tabl. 2). Èn fait quel que soit le lot, ces durées d'expulsion sont très variables. On peut le constater sur la figure 2 où est représentée chaque durée en fonction de la taille de la portée ; l'angle déterminé par les durées extrêmes n'est pas modifié par les différents traitements. Les temps moyens qui séparent les expulsions de deux porcelets sont aussi très variables, particulièrement après induction du cochonnage par A-PGF. Quant à l'ocytocine, son action n'est vraiment marquée que sur l'expulsion du deuxième et du cinquième fotus (fig. 3).

TABLEAU 2

Comparaison des durées d'expulsion des porcelets

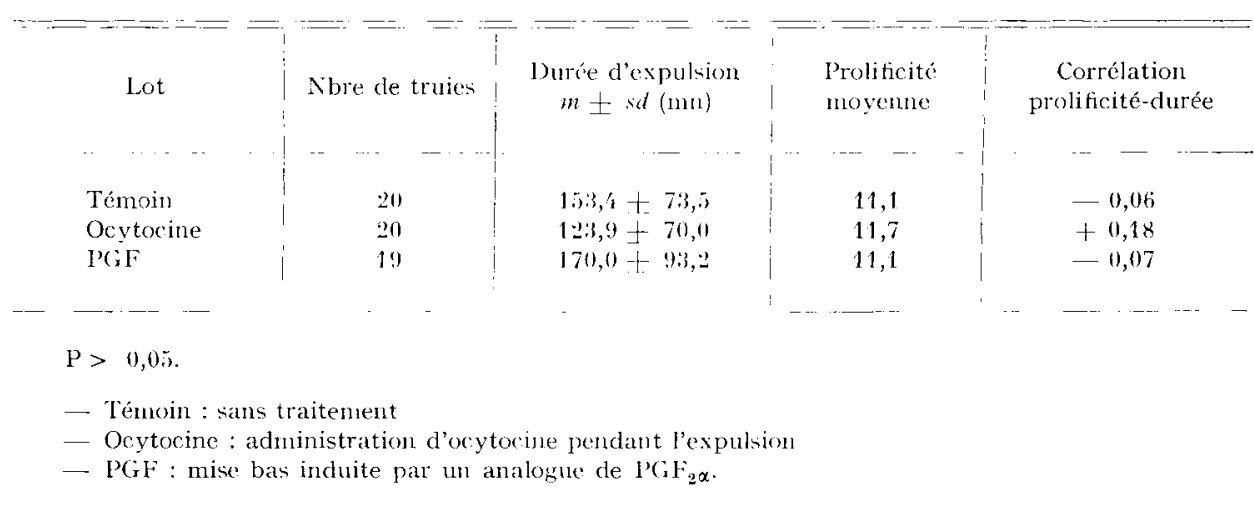

Le poids de naissance des porcelets est très différent $(P<0,0 I)$ suivant que le cochonnage a été induit ou non. Compte tenu de leur poids de naissance, la croissance moyenne des porcelets de lots $T$ et $O$ et des truies induites par A-PGF au III jour de la gestation n'est pas différente entre la naissance et le $2^{\mathrm{e}}$ jour de lactation, entre la naissance et le troisième jour et entre le troisième jour et le septième (fig. 4).

A la naissance, les taux de survie des porcelets sont indépendants du traitement 


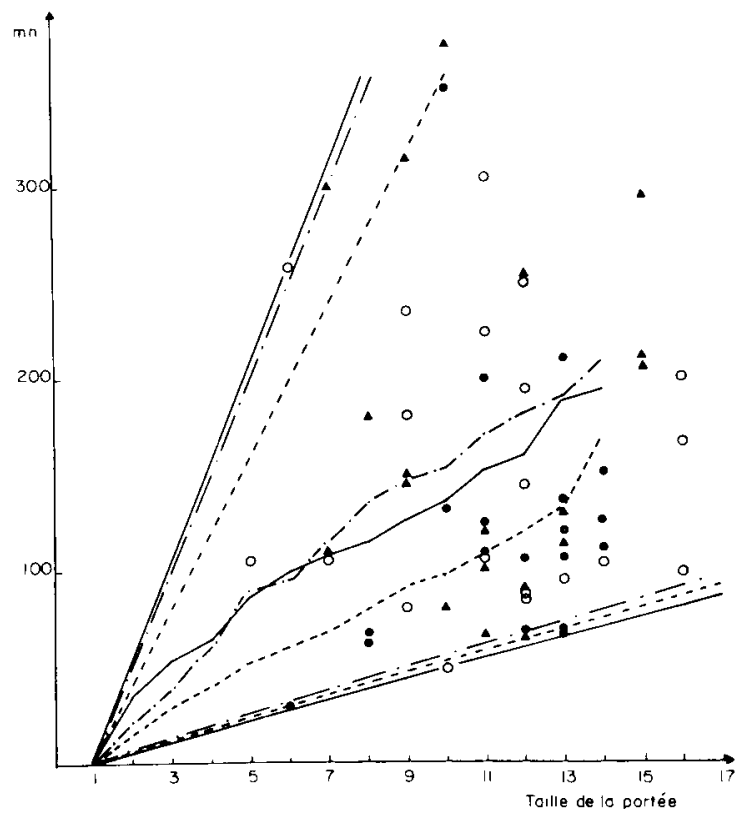

Fig. 2. - Durée totale et durée moyenne d'expulsion des porcelets suivant les traitements subis avant et pendant la mise bas

I.ot témoin : o duréc totale Lot $\mathrm{A}-\mathrm{PG}$ Lot $\mathrm{O}$
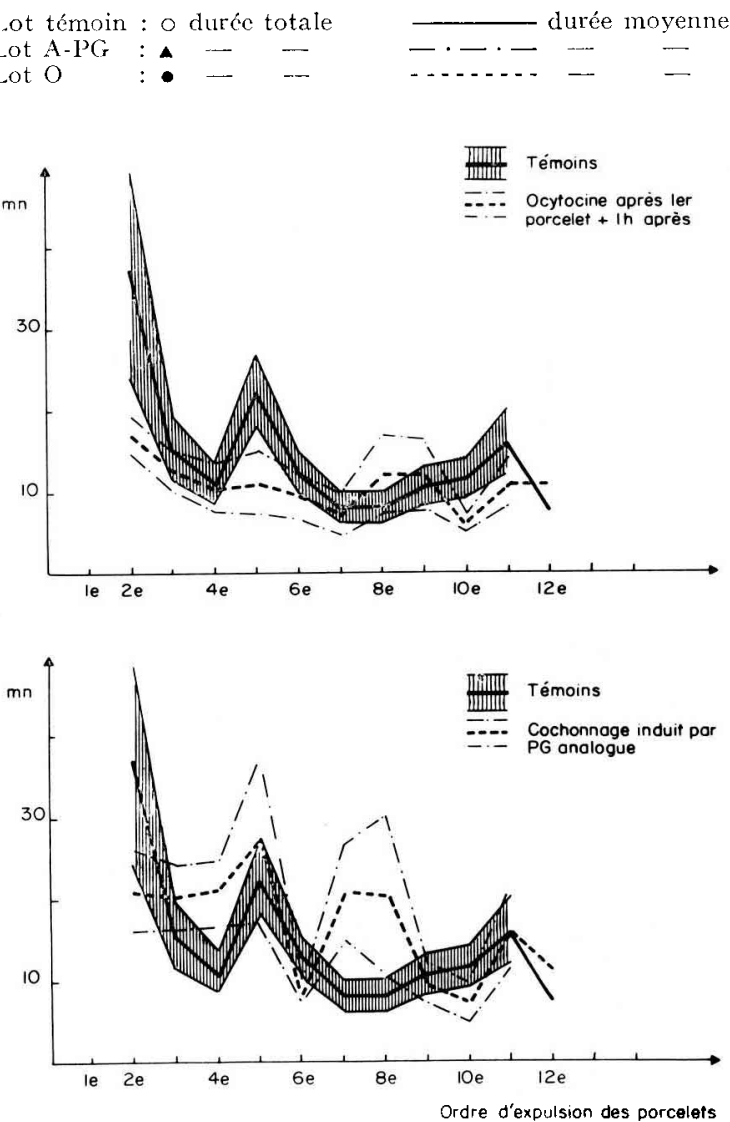

FrG. 3. - Intervalles entre l'expulsion de deux porcelets $(m \pm s m)$ Annales de Zootechnie. - I975. 


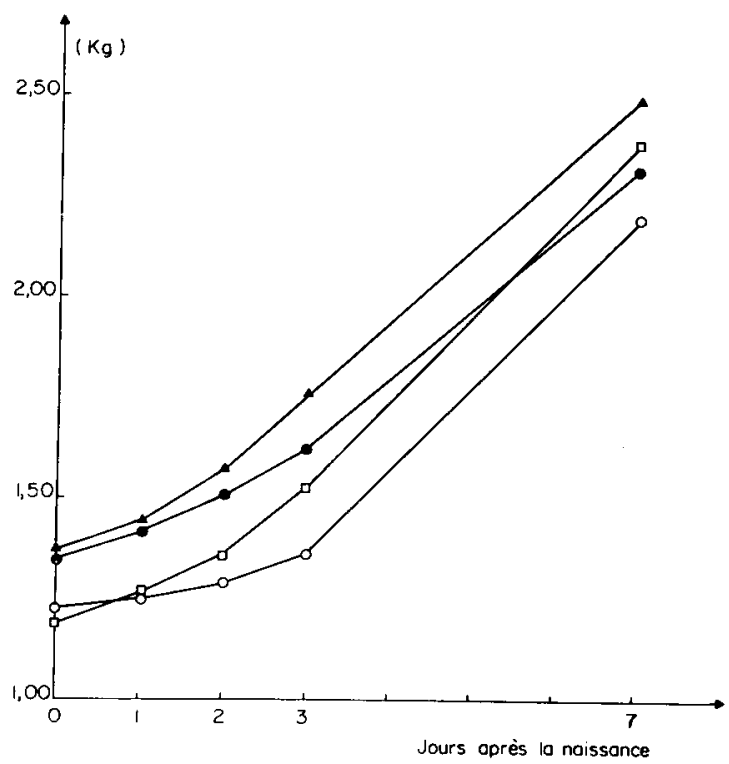

Iig. 4. - Evolution du poids moyen des porcelets au cours des 7 jou's suizant leur naissance

- Truies témoins

A Truies ayant reçu 2 administrations d'ocytocine au cours de l'expulsion

$\square$ Truies dont la mise bas a été induite par un analogue de $P_{G F} \alpha$ à GrII

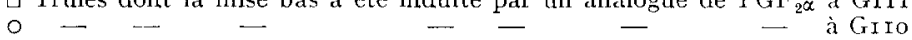

\section{TABLEAU 3}

Comparaison de la survie des porcelets suivant les traitements subis par les mères avant ou pendant la mise bas

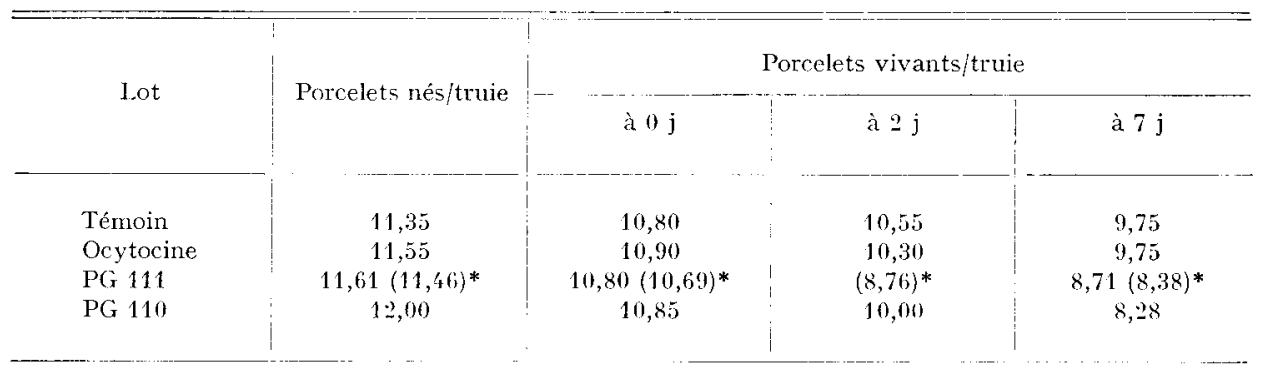

Témoin : sans traitement

Ocytocine : administrations d'ocytocine pendant l'expulsion

$\mathrm{PG}_{\mathrm{r}} 111$ : mise bas induite au $111^{\mathrm{e}}$ jour de gestation par analogue de $\mathrm{PGF}_{2 \alpha}$

$P G$ 110: mise bas induite au $110^{\circ}$ jour de gestation par un analogue de $\mathrm{PGF}_{2 \alpha}$

( ) * les observations ont été relevées au ¿e jour sur une partie des truies

Taux de survie différents entre les ${ }_{\mathrm{k}}$ lots : à $2 \mathrm{j}$ et $7 \mathrm{j}$

Taux de survie non différents de 2 à 7 j pour les lots $[$-O et $P G 111$. 
subi par la mère contrairement à ceux qui sont observés au $2^{\mathrm{e}}$ et au $7^{\mathrm{e}}$ jour de lactation $(\mathrm{P}<\mathrm{O}, \mathrm{OI}) \mathrm{Il}$ faut cependant remarquer qu'il n'y a pas de différences de mortalité entre le $2^{\mathrm{e}}$ et $7^{\mathrm{e}}$ jour entre les animaux des lots $\mathrm{T}$ et $\mathrm{O}$ et ceux qui sont nés après induction au III ${ }^{\mathrm{e}}$ jour de la gestation (tabl. 3).

Enfin après le sevrage, les truies sont venues en œstrus sans que nous puissions mettre en évidence une différence entre les lots.

\section{DISCUSSION}

Pour contrôler la mise bas, on peut soit prolonger la gestation jusqu'au moment désiré, soit induire la naissance avant qu'elle ne se produise normalement.

Chez la Truie, on peut aisément prolonger la gestation par administration de progestérone ou de progestagènes, ou par la formation de nouveaux corps jaunes en fin de gestation (Bosc, I974). On peut aussi utiliser le méthallibure ou dithiocarbamoylhydrazine (FIRST, I 972). En fait la prolongation de la gestation au-delà du terme s'accompagne d'une augmentation de la mortalité des porcelets à la naissance ce qui élimine cette façon de maîtriser le cochonnage (Bosc, I974).

La deuxième manière est d'induire la mise bas avant qu'elle ne se produise spontanément, et cette expérience montre les possibilités offertes par un analogue de $\mathrm{PGF}_{2 \alpha}$ (Binder et al., I974). Elle confirme les résultats de AsH et HEAP (I973) obtenus par injections successives d'un produit de même type, ou ceux de DIEHL. ct al. (I973) et de HENRICKs et HANDLIN (I974) avec les prostaglandines naturelles. Nos résultats indiquent de plus que l'intervalle "traitement-parturition », égal à $26 \mathrm{~h}$ en moyenne, dépend du moment de la gestation auquel l'administration d'APGF est faite, et aussi de la taille de la portée. Ces deux facteurs traduisent vraisemblablement l'influence des cestrogènes dans le sang maternel au moment du traitement. En effet leur taux plasmatique augmente rapidement pendant les derniers jours de la gestation, (TERQUI, I974 ; Robertson, et KING, I974) et leur biosynthèse est fœto-placentaire (FEVRE, LEGLISE, et RoMbaUts, I968; FEVRE, LEGLISE et REYNAUD, I972; FEVRe, I970). Cette influence des ostrogènes peut aussi rendre compte de l'importance de la production endogène de $\mathrm{PGF}_{2 \alpha}$ et par conséquent souligner son activité intrinsèque sur l'activité utérine. Cette prođuction de $\mathrm{PGF}_{2} \alpha$ en effet est contrôlée par les ostrogènes chez la Brebis (I,IGGIns et al., I973), ou chez la Chèvre gravide (Thorburn et al., I972). Pour cette dernière espèce, $\mathrm{PGF}_{2}$ a un rôle lutéolytique nécessaire au déroulement du travail (CuRRIE et ThorbURN, I973). Or chez la Truie le maintien de la gestation est assuré comme chez la Chèvre (Mristes et al., I95I) par la production de progestérone par les corps jaunes (du MeSNIL du Buisson et DaUzikr, I957) et le rôle lutéolytique de $\mathrm{PGF}_{2 \times}$ en fin de gestation a été montré par DIEHL et al. (I973) ou HENRICKs et HANDLIN (I974).

La dose de ioo $\mu \mathrm{g}$ de 1'analogue utilisé semble suffisante; il serait nécessaire cependant de le vérifier sur un plus grand nombre d'animatux. Quoiqu'il en soit avec $\check{5}^{\circ} \mathrm{O} \mu \mathrm{g}$ la variabilité des intervalles "traitement-parturition " est faible et dans cette expérience $90 \mathrm{p}$. Ioo des mise bas ont été groupées sur $\mathbf{I} 2 \mathrm{~h}$. Compte tenu de l'heure de traitement, on peut donc provoquer la majorité des mise bas pendant le temps normal de travail du personnel et modifier ainsi à son avantage la répartition circadienne des fréquences de mise bas. 
Les mise bas induites n'ont présenté aucun caractère particulier comme cela a déjà été noté (ASH et HEAP, I973; HeNricrs et HANdirin, I974). L,es durées d'expulsion des porcelets ont été légèrement plus longuej et les intervalles de naissance entre deux fœtus ont été plus variables que chez les témoins. Cette variabilité est normalement caractéristique de la Truie et elle a été souvent soulignée (voir Signoret, I970).

Nos observations montrent que la variabilité de la durée totale d'expulsion est peu affectée par administration d'ocytocine au cours du travail. Cette hormone dont l'action sur le myomètre est très rapide (ZFROBIN et SPORRI, I972), d'après nos observations, ne réduit que l'intervalle séparant l'expulsion des deux premiers porcelets et peut-être celui qui sépare le $4^{\mathrm{e}} \mathrm{du} 5^{\mathrm{e}}$.

Induire le part au $\mathrm{IIO}^{\mathrm{e}}$ ou $\mathrm{II}^{\mathrm{e}}{ }^{\mathrm{j}}$ jour de la gestation diminue significativenent le poids des porcelets à la naissance qui naissent alors 2 à 3 jours en moyenne avant les témoins. Compte tenu du poids de naissance, la croissance des porcelets nés le I $12^{c}$ jour n'a pas été affectée au cours des sept premiers jours de leur vie ce qui laisse supposer que la lactation des truies traitées le III jour de la gestation débute de façon satisfaisante. Il faut néanmoins relever une augmentation de mortalité postnatale qui se produit essentiellement au cours des deux jours qui suivent la mise bas. Cette augmentation peut être la conséquence du poids plus faible des porcelets nés après induction.

Cependant nos observations suggèrent aussi (qu'elle peut être attribuée au conportement maternel des truies, car le taux de porcelets écrasés par les truies induites par A-PGF est plus fort que dans les autres lots. Nous n'avons pas porté d'attention particulière au comportement maternel des truies et il est fort possible que l'induction de la mise bas par $\mathrm{PGF}_{2 \times}$ l'ait modifié comme cela a été remarqué chez la Lapine (Abis, Taurog et Nathanielsz, 1973).

In pratique, on peut en conclure que la mise bas ne doit pas être induite trop précocement et on peut penser que les possibilités offertes par les prostaglandines seront utilisées en particulier pour supprimer les mise bas qui se produisent en fin de semaine.

$$
\text { Resu pour publication on arril } 19 \pi 5 \text {. }
$$

\title{
REMERCIEMEN'TS
}

Cette étucle a été réalisée grâce à un financoment 1:. (). R. M. A. au titre d'une convention passée entre cet organisme, l'I. N. R. A. et l'I. 'T. P.

Nous remercions, par ailleurs, MIM. Salle, ('roczelle et MAupetit pour Ieur participation active à cette expérience.

\section{SUMMARY}

\author{
INDUCTION OF PARTURITION \\ IN THE SOW BY AN ANALOGUE OF PROSTAGIANDIN $\mathrm{F}_{2 x}$
}

Intramuscular injection of an analogue of Prostaglandin Ii $2 x$ (A-P'GF) (compound ICI $\left.n^{\circ} 80996\right)$ induces parturition in the sow. The "treatment-parturition $"$ interval lasted on an average $29 \mathrm{~h} 44( \pm 6 \mathrm{~h} 20$ ) and $25 \mathrm{~h} 24$ ( $\pm 3 \mathrm{~h} \mathrm{IO})$ when $\mathrm{I}_{5} \mathrm{O} \mu \mathrm{g}$ of A-P(iF were given on days 
I 10 and III, respectively of pregnancy. In the controls, the corresponding values were Ioo $h$ and $7^{6} \mathrm{~h}( \pm 35 \mathrm{~h})$. These intervals were related to prolificacy, the correlation being - 0.56 for all the sows treated. The duration of expulsion varied considerably, from I $53 \mathrm{mn}( \pm 73)$ in the control, it reached $170 \mathrm{mn}( \pm 93)$ after induction by A-PGF and was reduced to $124 \mathrm{mn}$ $( \pm 7$ ) when 2 injections of oxytocin were given after delivery of the first oestrus.

Birth weight was lower, and mortality during the first 2 days of life was higher in piglets of sows treated with A-PGF than in the controls. On the other hand, the growth of the piglets during the first week of lactation did not seem to be affected when $A-P G F$ was administrated on day I I of pregnancy.

\section{RÉFÉRENCES BIBLIOGRAPHIQUES}

Abel M., Talkog J., Nathanielsz P. W., i973. A comparison of the luteolytic effect of PG $F_{2 \alpha}$ and cortisol in the Pregnant Rabbit. Prostaglandins, 4, 43I-440.

Aiken J, W., I972. Aspirin and indomethacin prolong parturition in rats: evidence that prostaglandins contribute to expulsion of foetus. Vature (London), 240, $2 \mathrm{I}$.

Ash R. W., Heap R. B., I973. The induction and synchronization of parturition in sows treated with ICI 79939 an analogue of prostaglandin $\mathrm{F}_{2 \alpha}$. J. Agric. Sci., Camb., 81, 365-368.

Binder D., Bowler J., Brown E. D., Crossiey N. S., Hutron J., Senior M., Slater L., Wilkinson P., WRight N. C. A., I974, I6-Aryloxyprostaglandins : a new class of potent luteolytic agent. Prostaglandins, 6, 87-9o.

Bosc M. J., 1974. La parturition provoquće chez les mammifères domestiques. Avortement et Parturition provozués. Ed. Masson et Cie, Paris, I-24.

Bosc M., du Mesnil du Buisson I', Locatrlli A., i974. Mise en ćvidence d'un contrôle foetal de la parturition chez la Truie. Interaction avec la fonction lutéale. C. R. Acad. Sci. Paris, t. 278, Série D, I 507-1510.

Buckle J. W., Nathanielsz P. W., t973. The effect of low doses of Prostaglandins $F_{2 \alpha}$ infused into the aorta of uncestrained pregnant rats : observations on induction of parturition and effect on plasma progesterone concentration. Prostaglandins, 4, 443-455.

Brgicman M., Wiovist N., 1974. The use of Prostaglandins for the control of human fertility. Avortement et Panturition provoqués. Ed. Masson et Cie, Paris, I45-I62.

Challis J. R. G., Davies J. J., Ryan K. J., I973. The relationship between Progesterone and Prostaglandin $F$ concentrations in the plasma of pregnant rabbits. Prostaglandins, 4, 509-5i6.

Currie W. B., Thorburn G. D., I973. Induction of Premature Parturition in Goats by Prostaglandin $\mathrm{F}_{2 x}$ administered into the uterine vein Prostaglandins, 4, 20I-2I 4 .

Drint, J. R., Day 3. N., I974. Effect of Prostaglandin $F_{2 \alpha}$ on luteal function in swine. J. Anim. Sci, 37, p. $392-396$.

DiEht, J. R., Godke R. A., KILliax D. B., DAY B. N., I973. The induction of Parturition in Swine with Prostaglandin $\mathrm{F}_{2 \alpha}$. Soc. Stud. Reprod. 6th anmu. meet. Athens. U. S. A. abst. $\mathrm{n}^{\circ}$ I 10 .

Dunn M. V., Humphries N. G., Judins G. R., Kendall J. Z., Knight G. W., ig73. The effect of Prostaglandin $F_{2 x}$ antibody on gestation length in the rat. Prostaglandins, 3, 509-5I4.

Fevre J., r97o. Conversion en oestrone de quelques stéroides $C_{19}$ chez la Truie gestante. Ann. Biol. anim. Bioch. Biophys., 10, 25-33.

Fevre J., Leglise P. C., Rombauts P., ig68. Du rôle de l'hypophyse et des ovaires dans la biosynthèse des cestrogènes au cours de Ia gestation chez la Truie. Ann. Biol. anim. Bioch. Biophys., 8, 225-233.

Fevre J., Leglise P. C., Reynaud O., I972. Róle des surrénales maternelles dans la production. d'astrogènes par la Truie gravide. Ann. Biol. anim. Bioch. Biophys., 12, 559-567.

First N. L., I972. Inhibition of parturition in swine by the Dithiocarbamoylhydrazine ICI 33828. J. Reprod. Fent., 30, $321-323$.

Henricks D. M., Handlin D. L., I974. Induction of parturition in the sow with Prostaglandin $F_{2 \alpha}$. Theriogenology, 1, 7-I 4 .

Killian D. B., Garverick H. A., Day 13. N., 1972. Peripheral plasma progesterone and corticoid levels at parturition in the sow. J. Anim. Sci, 35, I I I (Abstr.)

Lliggins G. C., Fairclough R. J., Grieves S. A., Kendall J. Z., Knox B. S., ig73. The mechanism of initiation of Parturition in the Ewe. Rec. Progr. Horm. Res., 29, I I I-I59.

Meites J., Webster H. D., Young F. W., Thorp F., Hatch R. N., i95I. Effects of corpora lutea removal and replacement with progesterone on pregnancy in goats. J. A nim. Sci., 10, $4 \mathrm{Ir}$.

Du Mesnil du Ruisson F., Dauzier I., i957. Influence de l'ovariectomie chez la Truie pendant la gestation. Cple Rend. Sc. Soc. Biol., 61, 3 II.

Molokwe E. C. I., Waciner W. C., I973. Endocrine physiology of the puerperal sow. I. Anim. Sc: 35, I I $58-1$ I 63 . 
Nathaselsz P. W., Aber. M., Smth ( $x$. M., 1972. Initiation of parturition in the rabbit by intra aortic infusion of Prostaglandin $\mathrm{I}_{2} \alpha$. J. Lindocr., 55, 6r 7-6r8.

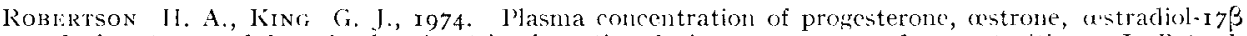
and of oestrone sulphate in the pig at implantation during pregnancy and at parturition. $J$. Reprod. Fivt. 40, I33-I4I.

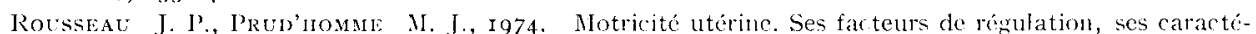
ristiques pendant la gestation et la parturition. Anortement et laarturition procoqués. Líd. Masson et Cie, Paris, $25-28$.

Sirinoret J. l', i970. Verhalten bei Schweinen in Verhalten Landwirt schaftlicher. Nutzicre. Berlin.

StabenteldT (i. H., r974. The role of progesterone in parturition : Premature, normal, prolonged gestation. Avortement et Parturition pronognés. Ed. Masson et Cie., l'aris, 97-1 22 .

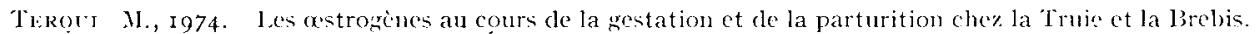
Avortenent et parlurition proroqués. Ė. Masson et Cie, Paris, 7I-79.

Tulkr M., I974. Blective indurtion of labour at term with oxytocin and Prostaglandins technique and fetal and maternal effact. Anortement et Parturition Procoques. Fid. Masson et Cie, Paris, $267-287$.

Thorbury (:. D., Nicol D. I., BAsset J. M., Shrtt J). A., Cox R. I., i972. Parturition in the goat and sheep: changes in corticosteroids, progesterone, oestrogens and prostaylandin Ji. J. Reprod. Fert., 16 suppl. 61-84.

Zerobin K., Spörra 1I., I972. Notility of the bovine and Porcine I'terus and the lablopian tube. Adi. Iet. Sci. and comp. Sci., 16, 303-3.t. 